\title{
Changes in Pramipexole Utilization after Introduction of the Extended-Release Formulation: A Nationwide Study in Taiwan
}

\author{
K. Arnold Chan ${ }^{1,2}$ (D) Yu-Chun Hsieh ${ }^{1} \cdot$ Shu-Feng Hsieh ${ }^{1} \cdot$ Rou-Shayn Chen $^{3}$
}

Accepted: 1 October 2020 / Published online: 16 October 2020

(c) The Author(s) 2020

\begin{abstract}
Background Real-world impact of extended-release formulations of oral drugs should ideally be evaluated in populationbased health data.

Objective To evaluate changes in utilization of the dopamine agonist pramipexole for Parkinson's disease after the introduction of extended-release (ER) pramipexole in Taiwan.

Patients and Methods Source data were derived from National Health Insurance claims. Patients with a diagnosis of Parkinson's disease and pramipexole prescriptions were identified. Drug use patterns from 2009 through 2011 (only the immediaterelease [IR] formulation was available) and from 2012 through 2017 (both the IR and ER formulations were available) were assessed. Outcomes of interest were levodopa equivalent dose per day (LEDD) and 1-year adherence, as measured by the medication possession ratio (MPR).

Results LEDDs associated with pramipexole ER prescriptions were more than twice as large as that associated with pramipexole IR, both in pramipexole used in monotherapy and that used in combination therapy. One-year MPRs for pramipexole ER initiators were all larger than 73\% from 2012 through 2016 and 1-year MPRs for pramipexole IR initiators were less than $65 \%$ in 2010 and 2011.

Conclusion Introduction of pramipexole ER to Taiwan resulted in higher LEDD in prescriptions with pramipexole. Patients with Parkinson's disease who were initiated on pramipexole ER had better adherence to the medication than those who were prescribed pramipexole IR.
\end{abstract}

\section{Background}

Evaluation of population-based drug-utilization data could provide insight into whether patients benefit from the best available treatment in the real-world setting. Among the many important topics of drug utilization research, assessing the use of an extended-release (ER) formulation of an oral

Electronic supplementary material The online version of this article (https://doi.org/10.1007/s40801-020-00215-6) contains supplementary material, which is available to authorized users.

K. Arnold Chan

kachan@ntu.edu.tw

1 Health Data Research Center, National Taiwan University, 33, Linsen South Road, Suite 526, Taipei, Taiwan

2 College of Medicine, National Taiwan University, 1 Jen-Ai Road, Section 1, Taipei, Taiwan

3 Division of Movement Disorders, Department of Neurology, Chang Gung Memorial Hospital and University at Linkou, 5, Fuxing Street, Guishan District, Taoyuan, Taiwan

\section{Key Points}

Introduction of extended-release pramipexole to Taiwan resulted in marked increase of its use over the following 5 years, but the concomitant decrease in immediaterelease pramipexole was not substantial over the same period.

Prescriptions with extended-release pramipexole were associated with higher levodopa equivalent dose per day than that for immediate-release pramipexole.

Patients who were initiated on extended-release pramipexole had better adherence, as measured by medication possession ratio, than those who were prescribed immediate-release pramipexole.

drug subsequent to introduction of the immediate-release (IR) formulation in a defined population would have major implications on clinical effectiveness and cost-effectiveness 
of the drug. ER formulations of oral drugs are associated with less frequent dosing and a more stable pharmacokinetic profile, resulting in more favorable treatment outcomes [1-3]. Due to the limited number of oral drugs with an ER formulation, this type of drug-utilization evaluation from a non-marketing perspective is rare in public-domain scientific journals. Use of ER methylphenidate was evaluated in a drug-utilization study of drugs for attention-deficit hyperactivity disorder (ADHD) in the UK, which was based on the population-based Clinical Practice Research Datalink [4]. While the use of IR and ER methylphenidate were described, it was not the main focus of the report, and there are few other population-based examples of utilization studies of oral drugs with an ER formulation.

Pramipexole is a non-ergot dopamine agonist developed for the treatment of Parkinson's disease [5]. The IR formulation was first approved for use in the USA in 1997 [6], and then received European Medicines Agency approval in 1998 [7]. The ER formulation was subsequently developed and was shown to be associated with better adherence to the drug regimen, more constant blood level, and favorable clinical response and safety profiles in clinical trials [8-11]. Investigators from Italy studied Parkinson's disease patients from a single center in Rome and reported that the use of ER dopamine agonists, including transdermal rotigotine and ER formulations of oral ropinirole and pramipexole, was associated with lower daily levodopa doses and decreased frequency of adverse effects [12]. It would be valuable if the change in drug use patterns could be evaluated at a population level in different countries with different ethnic compositions and clinical practices.

In 2011, pramipexole ER was approved for use in Taiwan, a country with a national health-insurance program that captures all drug-use information for insurance-covered prescription drugs. With this population-based data source we carried out a study to evaluate the use of all formulations of pramipexole (IR and ER) before and after the ER formulation was introduced. We evaluated whether the use of pramipexole ER was associated with higher dopamine agonist doses (represented by the levodopa equivalent dose [LED]) and better adherence among Parkinson's disease patients in Taiwan. The LED was proposed by Tomlinson and colleagues in 2010, and is the dose of an anti-Parkinson drug that would achieve the same treatment effect as $100 \mathrm{mg}$ of levodopa combined with a dopaminedecarboxylase inhibitor, and this unit is suitable for comparing dose strengths across different anti-Parkinson drugs [13].

\section{Methods}

\subsection{Data Source}

The publicly funded and compulsory national health insurance (NHI) program in Taiwan started in 1995 and provides coverage for clinical services and prescription drugs. The de-identified NHI data are available for research through the Department of Statistics, Ministry of Health and Welfare [14-16]. Disease diagnoses in NHI were coded according to the International Classification of Diseases, Ninth Revision, Clinical Modifications (ICD-9-CM) system until 2015 and then switched to the Tenth Revision (ICD-10-CM) in 2016. Individual drug products are assigned specific codes by the NHI Administration for reimbursement purposes, and procedures have been developed to map the codes to the World Health Organization Anatomical Therapeutic Chemical (ATC) classification system for specific drugs [17].

\subsection{Study Data and Study Design}

NHI data from 2009 through 2017 were utilized for this retrospective drug-utilization study. Data included demographic information, diagnosis codes associated with outpatient visits, emergency room visits and hospitalizations, and drug prescriptions. The study was exempted from ethics review because de-identified data were utilized (National Taiwan University Hospital Research Ethics Committee approval document 201901080W).

The study population included those who had at least one pramipexole prescription and diagnosis code for Parkinson's disease (ICD-9-CM 332.0 or ICD-10-CM G20) from 2009 through 2017 . The disease code eligibility was occurrence of two diagnosis codes associated with outpatient or emergency visits on different days within a 1-year period or a diagnosis code associated with one hospital discharge. There was no restriction on the temporal sequence or number of days between the pramipexole prescription and the disease code. Those who were younger than 20 years at the time of the first pramipexole prescription during the study period were excluded.

Pramipexole at doses of $0.125 \mathrm{mg}, 0.25 \mathrm{mg}$, and $1 \mathrm{mg}$ per tablet received market approval in Taiwan in 2001 and all of them were of the IR formulation. They were listed for NHI reimbursement in 2002 and the generic versions began to be listed for NHI coverage in 2013. Different doses of pramipexole ER $(0.375 \mathrm{mg}, 0.75 \mathrm{mg}, 1.5 \mathrm{mg}, 2.25 \mathrm{mg}, 3 \mathrm{mg}, 3.75 \mathrm{mg}$, and $4.5 \mathrm{mg}$ per tablet) were approved in August 2010 and June 2011; they were listed for NHI reimbursement on 1 July 2011. In this study all pramipexole products (brand name IR, generic IR, and brand name ER) were the drugs of interest and all pramipexole prescriptions among study subjects were evaluated. Pramipexole prescriptions included pramipexole as monotherapy and pramipexole with other anti-Parkinson drug(s) as combination therapy. The study design is a beforeand-after comparison of pramipexole use, covering a period when only pramipexole IR was available and the following period when both pramipexole IR and pramipexole ER were available in a defined population. 


\subsection{Time Periods and Outcomes}

According to the time of introduction of pramipexole ER to the Taiwan market, the two periods of interest were 2009 through 2011 and 2012 through 2017, as there was negligible pramipexole ER prescription in 2011. All anti-Parkinson drug prescriptions among the study subjects from 2009 through 2017 were assessed. In addition to pramipexole, they were pharmacologically grouped into levodopa (single-ingredient and combination products), catechol-o-methyl transferase inhibitor, ergot dopamine agonists, non-ergot dopamine agonists, monoamine oxidase type B inhibitors, amantadine, and anticholinergics (Table 1).

The two major outcome metrics of interest were LED per prescription per day (LEDD) and adherence to pramipexole after the initial prescription. LED is the dose of an anti-Parkinson drug that would achieve the same treatment effect as $100 \mathrm{mg}$ of levodopa combined with a dopaminedecarboxylase inhibitor. LEDs for anti-Parkinson drugs other than anticholinergics, as described by Tomlinson and colleagues, are listed in Table 1 [13]. LEDD is the combined daily LED for all anti-Parkinson drugs in the same prescription. LEDDs were only calculated for outpatient prescriptions with pramipexole. For Parkinson's disease patients, the medication possession ratio (MPR) was the metric that represented adherence to pramipexole during 1 year after the initial pramipexole prescription [18].

\subsection{Statistical Analysis}

In this study there were two units of analysis-individual subjects and individual prescriptions with pramipexole. For all study subjects, gender and age at the time of first eligible Parkinson's disease diagnosis after 1 January 2009 were described. Annual number of prescriptions for pramipexole IR, pramipexole ER and other anti-Parkinson drugs were compiled for the study subjects from 2009 through 2017, and analysis was restricted to prescriptions associated with outpatient visits. The specialty of the physician who prescribed pramipexole at the outpatient clinic was assessed. The small number of prescriptions with both pramipexole IR and pramipexole ER (less than $1 \%$ of the total) were excluded from analyses comparing pramipexole IR prescriptions and pramipexole ER prescriptions. The proportion of all outpatient anti-Parkinson prescriptions with pramipexole and proportion of all dopamine agonist prescriptions with pramipexole were calculated for each year during the study period. The proportion of pramipexole ER initiators who had no prior pramipexole IR prescription since 1 January 2009 were assessed.

LEDD for each pramipexole prescription was assessed by first summing the LED for each anti-Parkinson drug in the same prescription, then the total LED of the prescription was divided by the days' supply of the prescription, resulting in the LEDD. For a prescription with drugs for different durations of use, the days' supply of the drug with the longest duration of use was treated as the days' supply for the whole prescription. For example, for a prescription with a drug for 28 days and another drug for 7 days, LEDD for the prescription was the sum of the LEDs associated with each drug divided by 28. Pramipexole-specific LEDD and total LEDD was evaluated for all pramipexole prescriptions. Annual average pramipexole-specific LEDD for all pramipexole prescriptions, stratified by pramipexole as monotherapy and combination therapy for IR and ER formulations, was calculated for each year during the study period. For each prescription of pramipexole as part of a combination regimen, the proportion of total LEDD contributed by pramipexole was assessed, and the annual average of proportion of LEDD contributed by pramipexole in these prescriptions was evaluated for each year during the study period.

Adherence to pramipexole was evaluated on a per-patient basis. Patients who were prescribed pramipexole after 1 January 2010 and had at least 180 days of look-back period to evaluate incident prescription status were eligible. Adherence evaluation was restricted to new users of pramipexole, operationally defined as no prior use of pramipexole within the previous 180 days, and those who died within 1 year after pramipexole initiation were excluded. One-year pramipexole MPR for a patient was calculated by summing the days' supply for the pramipexole prescriptions of interest (IR or ER) regardless of potential overlapping days within 1 year after the initiating prescription and dividing that total days' supply by 365 . Average 1-year MPRs for all pramipexole IR and ER initiators during each year from 2010 through 2016 were evaluated.

\section{Results}

\subsection{Study Subjects}

Within the Taiwan NHI research data environment, 35,698 subjects had a diagnosis code of Parkinson's disease and at least one pramipexole prescription from 2009 through 2017. Less than 5\% were younger than 50 years and more than $60 \%$ were between 61 and 80 years of age (Table 2).

Excluding 49 patients whose only pramipexole prescriptions were associated with emergency room visits or hospitalizations, the study subjects received 931,819 pramipexole prescriptions associated with outpatient visits during the study period. Number of prescriptions for different antiParkinson drugs among the 35,649 patients in each year from 2009 through 2017 are given in Table 3. Neurologists prescribed $88 \%$ of the pramipexole prescriptions. In Table 3 a prescription of combination therapy could appear in multiple categories. Slightly less than $1 \%$ of prescriptions (9112) 
Table 1 Anti-Parkinson drugs available in Taiwan and levodopa equivalent dose

\begin{tabular}{|c|c|c|c|}
\hline Drug class & Drug name & $\begin{array}{l}\text { Anatomical therapeutic } \\
\text { chemical category }\end{array}$ & $\begin{array}{l}\text { Levodopa equivalent } \\
\text { dose (mg/100 mg } \\
\text { levodopa) }\end{array}$ \\
\hline \multirow[t]{4}{*}{ Levodopa } & Levodopa & N04BA01 & $\begin{array}{l}100 \text { (immediate release) } \\
133 \text { (controlled release) }\end{array}$ \\
\hline & Levodopa + Benserazide & N04BA02 & 100 \\
\hline & Levodopa + Carbidopa & N04BA02 & 100 \\
\hline & Levodopa + Carbidopa + Entacapone & N04BA03 & 75 \\
\hline Catechol-O-methyl transferase inhibitors & Entacapone & N04BX02 & Levodopa dose $\times 0.33^{\mathrm{a}}$ \\
\hline \multirow[t]{7}{*}{ Dopamine agonist } & $\begin{array}{l}\text { Bromocriptine } \\
\text { (ergot dopamine agonist) }\end{array}$ & G02CB01 & 10 \\
\hline & $\begin{array}{l}\text { Lisuride } \\
\text { (ergot dopamine agonist) }\end{array}$ & G02CB02 & 1 \\
\hline & $\begin{array}{l}\text { Pergolide } \\
\text { (ergot dopamine agonist) }\end{array}$ & N04BC02 & 1 \\
\hline & Ropinirole & N04BC04 & 5 \\
\hline & Pramipexole & N04BC05 & 1 \\
\hline & Apomorphine & N04BC07 & 10 \\
\hline & Rotigotine & N04BC09 & 3.3 \\
\hline \multirow[t]{2}{*}{ Monoamine oxidase type B inhibitors } & Selegiline & N04BD01 & $\begin{array}{l}10 \text { (oral) } \\
1.25 \text { (sublingual) }\end{array}$ \\
\hline & Rasagiline & N04BD02 & 1 \\
\hline Amantadine & Amantadine & N04BB01 & 100 \\
\hline \multirow[t]{4}{*}{ Anticholinergics } & Trihexyphenidyl & N04AA01 & Not applicable \\
\hline & Biperiden & N04AA02 & Not applicable \\
\hline & Piroheptine & N04AA91 & Not applicable \\
\hline & Benztropine (Benzatropine) & N04AC01 & Not applicable \\
\hline
\end{tabular}

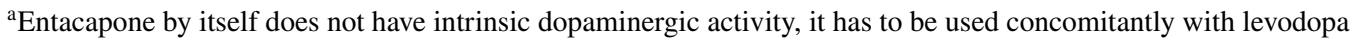

had both pramipexole IR and pramipexole ER in the same prescription.

Pramipexole IR and ER were mostly used in combination therapy with other anti-Parkinson drugs in outpatient settings in Taiwan (Fig. 1 and Supplementary Table 1, Online Supplementary Material (OSM)). Throughout the study period $89.1 \%$ of pramipexole IR prescriptions were combination therapy and $94.3 \%$ for pramipexole ER. Pramipexole IR use in combination therapy was increasing during the 3 years before introduction of the ER formulation, and the increasing trend was reversed afterwards. Pramipexole ER prescriptions in monotherapy and combination therapy increased by tenfold from 2012 through 2017, while the use of pramipexole IR in either monotherapy or combination therapy did not decrease substantially during the same period.

Distributions of combination regimens with pramipexole before and after pramipexole was introduced are given in Table 4, with levodopa as the most common drug used in combination with pramipexole IR and pramipexole ER. The median dose of pramipexole IR was $0.5 \mathrm{mg}$ for monotherapy and $0.75 \mathrm{mg}$ for combination therapy, which did not change after pramipexole ER was introduced (Table 5). The median dose of pramipexole ER was higher than that for pramipexole IR for both monotherapy and combination therapy. Among 12,757 pramipexole ER initiators, 4101 (32.1\%) had no prior pramipexole IR prescription since 1 January 2009.

For pramipexole as a proportion of all anti-Parkinson prescriptions among the study subjects, the secular trend was similar to that shown in Fig. 1 after the introduction of pramipexole ER (Fig. 2 and Supplementary Table 2, OSM). Pramipexole ER prescriptions as a proportion of all

Table 2 Patients with Parkinson's disease diagnosis and pramipexole prescription, 2009-2017

\begin{tabular}{lrrr}
\hline $\begin{array}{l}\text { Age } \\
\text { (years) }\end{array}$ & \multicolumn{1}{l}{ Male } & \multicolumn{1}{l}{ Female } & \multicolumn{1}{l}{ Total } \\
\hline $20-50$ & $942(5.0 \%)$ & $654(3.8 \%)$ & $1596(4.5 \%)$ \\
$51-60$ & $2750(14.7 \%)$ & $2337(13.7 \%)$ & $5087(14.3 \%)$ \\
$61-70$ & $5193(27.8 \%)$ & $4881(28.6 \%)$ & $10,074(28.2 \%)$ \\
$71-80$ & $6432(34.5 \%)$ & $6583(38.6 \%)$ & $13,015(36.5 \%)$ \\
$\geq 81$ & $3338(17.9 \%)$ & $2588(15.2 \%)$ & $5926(16.6 \%)$ \\
Total & $18,655(52.3 \%)$ & $17,043(47.7 \%)$ & $35,698(100.0 \%)$ \\
\hline
\end{tabular}


anti-Parkinson prescriptions increased steadily from less than $3 \%$ in 2012 to more than $26 \%$ in 2017 , and the proportion for pramipexole IR decreased from 35.7 to $20.7 \%$ during the same period. Proportions of all dopamine agonist prescriptions with pramipexole ER increased from $5.1 \%$ in 2012 to $42.3 \%$ in 2017 , and the same proportions for pramipexole IR decreased from 65.2 to $33.2 \%$ over the same period.

For all pramipexole prescriptions, average LEDD per prescription was higher for pramipexole ER monotherapy and combination therapy prescriptions than that for pramipexole IR prescriptions (Fig. 3 and Supplementary Table 3, OSM). For pramipexole monotherapy, average LEDD per prescription was 72.7 from 2009 through 2011 when only pramipexole IR was available; LEDD per prescription for pramipexole ER monotherapy was greater than 100 in all years from 2012 through 2017. For pramipexole used in combination therapy, average LEDD per prescription for pramipexole IR was 132.9 from 2009 through 2011 and the average LEDD per prescription was more than 150 for pramipexole ER from 2012 through 2017. Average proportion of LEDD contributed by pramipexole IR in combination regimens was $24.4 \%$ from 2009 through 2011 and it was $30.7 \%$ for pramipexole ER from 2012 through 2017 (Fig. 4 and Supplementary Table 4, OSM).

One-year MPRs for pramipexole IR initiators were less than 65\% in 2010 and 2011 (Fig. 5 and Supplementary Table 5, OSM). For pramipexole ER initiators, 1-year MPRs were all larger than 73\% from 2012 through 2016.

\section{Discussion}

To the best of our knowledge this is one of very few population-based studies that evaluated the utilization patterns of all formulations of a drug after the ER formulation was introduced. The before-and-after comparison has provided valuable information on utilization of the ER version and

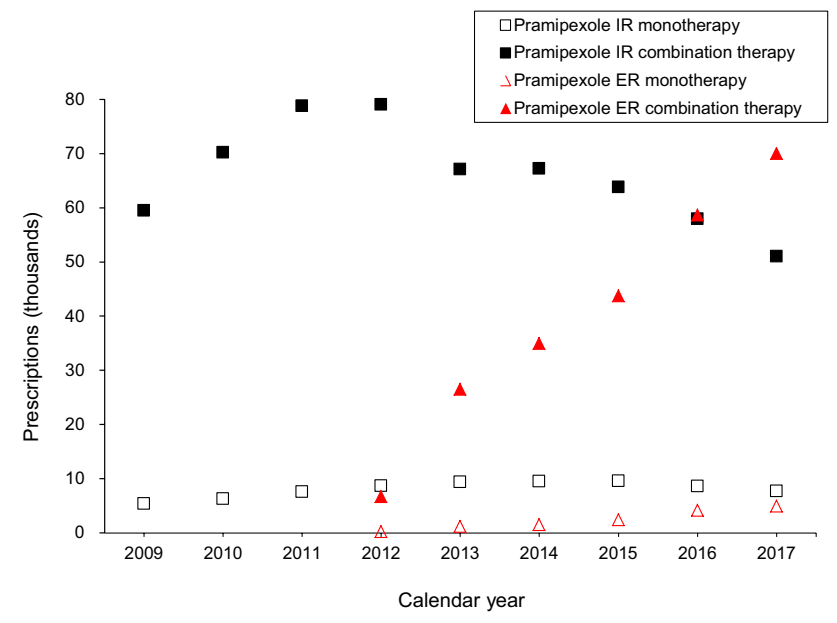

Fig. 1 Annual number of pramipexole prescriptions, 2009-2017

the concurrent changes in the use of the IR version. The findings could serve as the basis for further evaluation of the effectiveness, safety and budget impact of the drug when used in a real-world setting.

Aside from the methodology implications described above, the study has provided insight into the use of pramipexole for Parkinson's disease in Taiwan. The potential impact of pramipexole ER introduction was represented by two pre-defined metrics, LEDD and adherence. Pharmacologically higher LEDDs would result in stronger anti-Parkinson effects and better adherence would lead to better treatment outcomes. With the more convenient dosing schedule, it was anticipated that the use of the ER formulation would lead to improvement in both measures and the hypothesis was strongly supported by the empirical prescription data. Not being a clinical study with primary data collection from individual patients, at population level the data showed that those who received pramipexole ER had a higher LEDD and better adherence

Table 3 Anti-Parkinson prescriptions in outpatient settings among 35,649 patients, ${ }^{\mathrm{a}} 2009-2017^{\mathrm{b}}$

\begin{tabular}{lrrrrrrrrrr}
\hline & 2009 & 2010 & 2011 & 2012 & 2013 & 2014 & 2015 & 2016 & 2017 & Total \\
\hline Levodopa & 144,838 & 166,022 & 185,907 & 205,363 & 224,737 & 238,285 & 247,840 & 251,947 & 244,517 & $1,909,456$ \\
Catechol-O-methyl transferase inhibitors & 26,674 & 29,705 & 33,706 & 35,181 & 36,876 & 37,679 & 37,257 & 36,666 & 35,651 & 309,395 \\
Dopamine agonist & & & & & & & & & & \\
Pramipexole immediate-release & 64,930 & 76,533 & 86,394 & 88,049 & 77,881 & 78,561 & 75,446 & 68,621 & 60,373 & 676,788 \\
Pramipexole extended-release & & & & 7233 & 28,981 & 38,287 & 48,184 & 64,862 & 76,596 & 264,143 \\
All other dopamine agonists & 25,110 & 28,274 & 34,051 & 41,877 & 48,545 & 49,833 & 50,932 & 47,513 & 44,198 & 370,333 \\
Monoamine oxidase type B inhibitors & 15,761 & 16,354 & 17,260 & 18,900 & 19,635 & 19,064 & 21,440 & 23,182 & 23,165 & 174,761 \\
Amantadine & 49,962 & 55,131 & 60,641 & 66,026 & 71,274 & 76,374 & 77,416 & 79,211 & 78,197 & 614,232 \\
Anticholinergics & 58,586 & 63,927 & 68,223 & 72,316 & 75,155 & 75,987 & 74,020 & 73,526 & 70,137 & 631,877 \\
\hline
\end{tabular}

a49 subjects only had inpatient or emergency room prescription records and they did not have prescriptions relevant to this table

${ }^{\mathrm{b}}$ The counts are not mutually exclusive, as one prescription with multiple drugs would be counted under more than one category 
Table 4 Distribution of combination regimen prescriptions with pramipexole, 2009-2017

\begin{tabular}{|c|c|c|}
\hline Combination regimen & $\begin{array}{l}2009-2011 \\
\text { Number of pre- } \\
\text { scriptions (\%) }\end{array}$ & $\begin{array}{l}2012-2017 \\
\text { Number of pre- } \\
\text { scriptions (\%) }\end{array}$ \\
\hline Pramipexole IR + LD & $66,199(31.8)$ & $159,242(41.3)$ \\
\hline Pramipexole IR + LD + COMTi & $27,393(13.1)$ & $37,818(9.8)$ \\
\hline Pramipexole IR + LD + anticholinergics & $23,594(11.3)$ & $50,479(13.1)$ \\
\hline Pramipexole IR + LD + amantadine & $23,313(11.2)$ & $44,225(11.5)$ \\
\hline Pramipexole IR + LD + COMTi + amantadine & $13,494(6.5)$ & $14,996(3.9)$ \\
\hline Pramipexole IR $+\mathrm{LD}+$ amantadine + anticholinergics & $8598(4.1)$ & $13,376(3.5)$ \\
\hline Pramipexole IR + LD + COMTi + anticholinergics & $7927(3.8)$ & $10,998(2.9)$ \\
\hline Pramipexole IR + anticholinergics & $4976(2.4)$ & $11,604(3.0)$ \\
\hline Pramipexole IR + LD + MAOBi & $4311(2.1)$ & $6763(1.8)$ \\
\hline Pramipexole IR + LD + COMTi + amantadine + anticholinergics & $4135(2.0)$ & $5234(1.4)$ \\
\hline Pramipexole IR + amantadine & $4126(2.0)$ & $9210(2.4)$ \\
\hline Pramipexole IR + LD + COMTi + MAOBi & $2210(1.1)$ & $1441(0.4)$ \\
\hline Pramipexole IR + MAOBi & $1806(0.9)$ & $2914(0.8)$ \\
\hline Others & $16,249(7.8)$ & $17,344(4.5)$ \\
\hline Total & $208,331(100)$ & $385,644(100)$ \\
\hline Pramipexole ER + LD & & $71,306(29.7)$ \\
\hline Pramipexole ER + LD + amantadine & & $34,084(14.2)$ \\
\hline Pramipexole ER + LD + COMTi & & $29,496(12.3)$ \\
\hline Pramipexole ER + LD + anticholinergics & & $24,819(10.3)$ \\
\hline Pramipexole ER + LD + COMTi + amantadine & & $20,408(8.5)$ \\
\hline Pramipexole ER + LD + amantadine + anticholinergics & & $10,375(4.3)$ \\
\hline Pramipexole ER + LD + COMTi + anticholinergics & & $8251(3.4)$ \\
\hline Pramipexole ER + LD + MAOBi & & $6296(2.6)$ \\
\hline Pramipexole ER + LD + MAOBi + amantadine & & $5573(2.3)$ \\
\hline Pramipexole ER + LD + COMTi + amantadine + anticholinergics & & $5133(2.1)$ \\
\hline Pramipexole ER + anticholinergics & & $4747(2.0)$ \\
\hline Pramipexole ER + amantadine & & $3692(1.5)$ \\
\hline Others & & $16,271(6.8)$ \\
\hline Total & & $240,451(100)$ \\
\hline
\end{tabular}

$I R$ immediate release, $E R$ extended release, $L D$ levodopa, $C O M T i$ catechol-o-methyl transferase inhibitors, $M A O B i$ monoamine oxidase type B inhibitor

Table 5 Pramipexole dosage in outpatient prescriptions, 2009-2017

\begin{tabular}{|c|c|c|c|c|}
\hline & \multicolumn{4}{|c|}{ Dose per prescription } \\
\hline & \multicolumn{2}{|l|}{ 2009-2011 } & \multicolumn{2}{|l|}{ 2012-2017 } \\
\hline & Median (mg) & $\begin{array}{l}\text { Interquartile } \\
\text { range (mg) }\end{array}$ & Median (mg) & $\begin{array}{l}\text { Interquartile } \\
\text { range (mg) }\end{array}$ \\
\hline Pramipexole immediate-release in monotherapy regimen & 0.5 & $0.25-0.75$ & 0.5 & $0.25-0.75$ \\
\hline Pramipexole immediate-release in combination therapy regimen & 0.75 & $0.5-1.75$ & 0.75 & $0.375-0.75$ \\
\hline Pramipexole extended-release in monotherapy regimen & & & 0.75 & $0.375-1.5$ \\
\hline Pramipexole extended-release in combination therapy regimen & & & 1.5 & $0.75-3$ \\
\hline
\end{tabular}




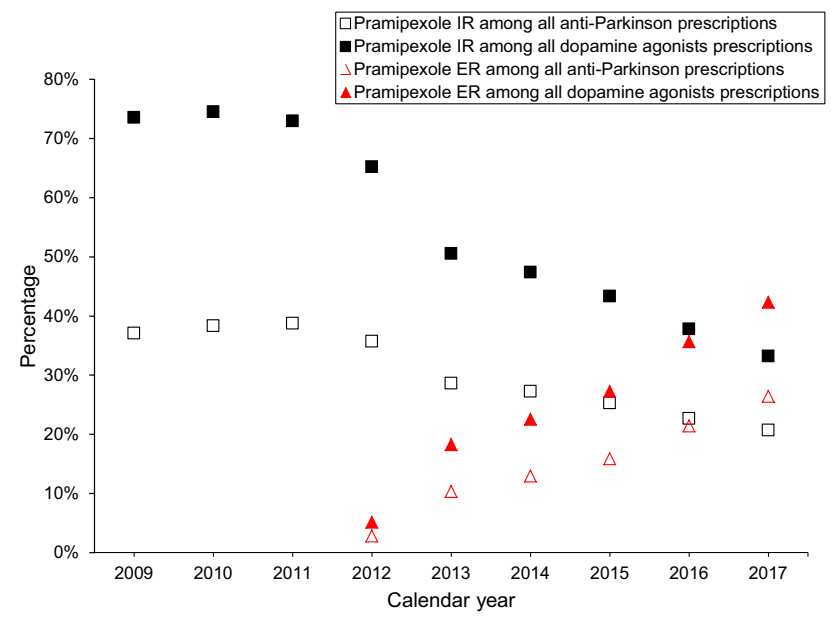

Fig. 2 Proportion of anti-Parkinson prescriptions and proportion of dopamine agonists prescriptions with pramipexole, 2009-2017

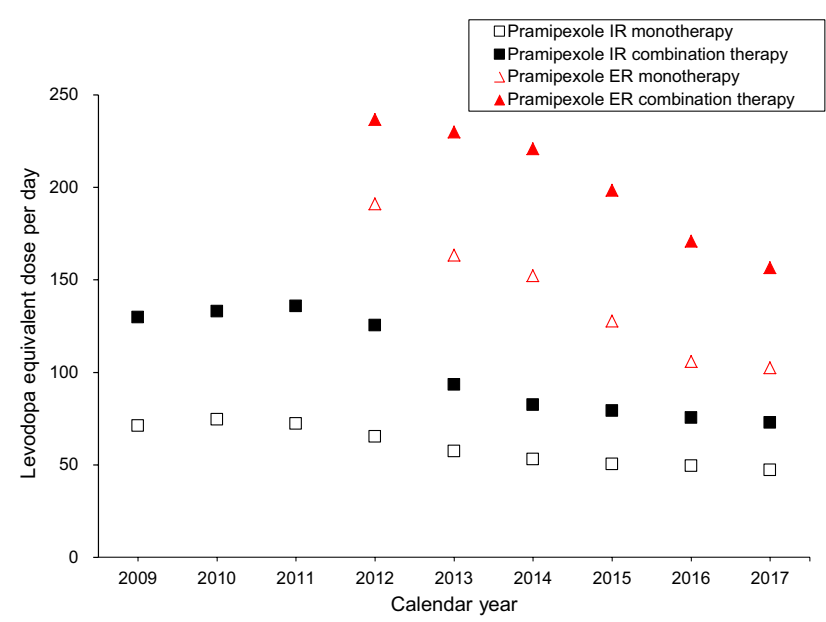

Fig. 3 Levodopa equivalency dose per day (LEDD) per prescription for prescriptions with pramipexole, 2009-2017

than those who received pramipexole IR during the same period. In an eight-center study of 112 Parkinson's disease patients in Europe, patients with adherence better than $80 \%$ experienced clinically better motor outcomes [19]. While no similar correlation between adherence and clinical outcomes has been demonstrated in Taiwan, in all likelihood higher LEDD and better adherence would result in favorable anti-Parkinson outcomes after the introduction pramipexole ER in Taiwan.

Pellicano and colleagues reported the impact of ER dopamine agonists among patients with early Parkinson's disease from a single specialty clinic in a hospital in Rome over two 12-month periods, with 56 patients in 2007 and 72 patients in 2011 [12]. The investigators reported no significant difference in terms of treatment outcomes, as measured by the

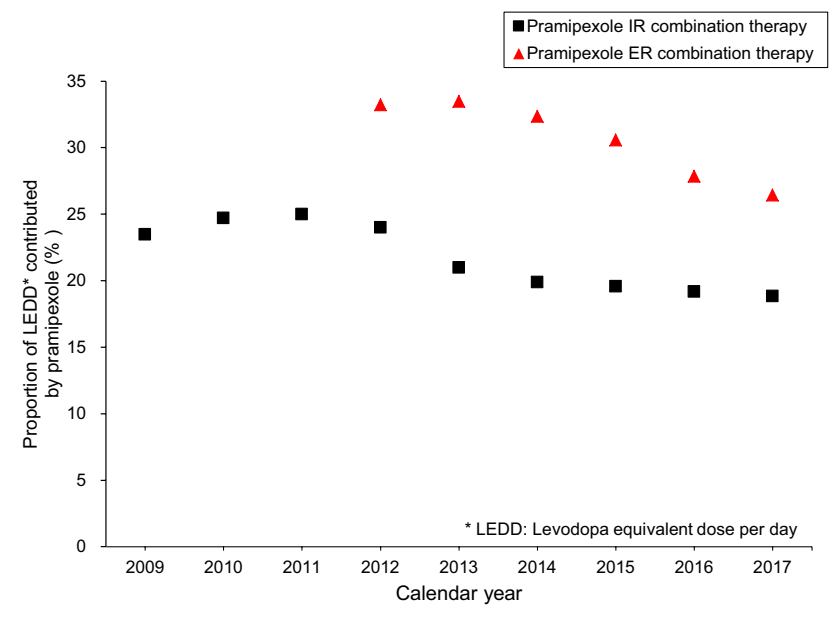

Fig. 4 Percentage of levodopa equivalency dose per day (LEDD) contributed by pramipexole, 2009-2017

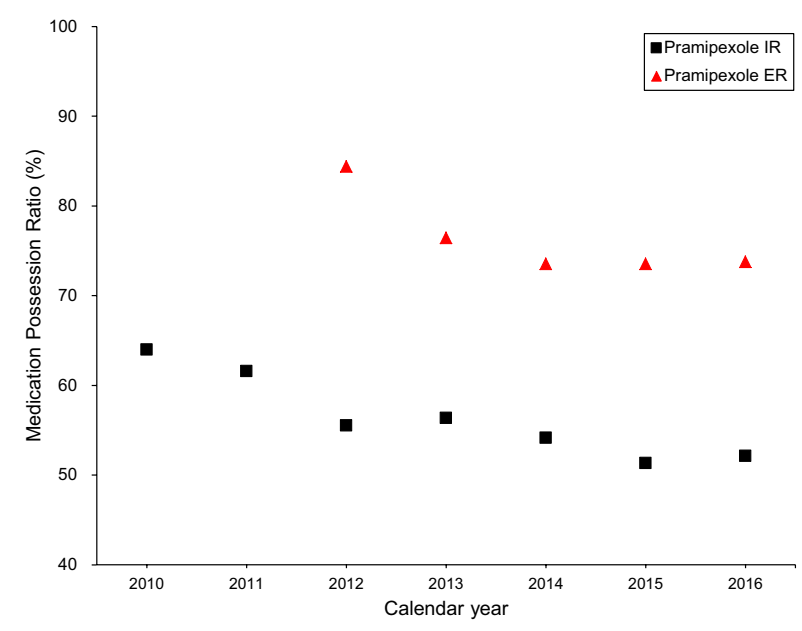

Fig. 5 Medication possession ratio among pramipexole initiators

Unified Parkinson's Disease Rating Scale part III score, between the two periods and a significant decrease in frequency of adverse drug effects. Due to the limited scope of our study, we could not evaluate clinical outcomes with regard to beneficial and adverse effects as a result of antiParkinson drugs, as clinical outcomes could not be reliably ascertained through health insurance claims data without information on neurologic and radiologic examinations. The study from Rome included only patients with early Parkinson's disease, and our study included Parkinson's disease patients of all levels of severity and duration. An increase in dopamine agonist monotherapy among patients aged 65-75 years was observed among the Italian patients when the ER dopamine agonists became available in 2011, and we observed different usage patterns of pramipexole in Taiwan.

When pramipexole ER became available in Taiwan, use of pramipexole IR decreased afterwards, but not substantially. 
Availability of a generic version of pramipexole IR would not adequately explain this prescription pattern, as there was no substantial co-payment difference between brand name and generic pramipexole IR under the Taiwan NHI. A more likely explanation is the clinical inertia phenomenon that is commonly observed in the management of chronic diseases without apparent symptoms [20]. Whether the same phenomenon applies to Parkinson's disease, a neurologic condition associated with apparent symptoms, has not been adequately evaluated. It was likely that some Parkinson disease patients in Taiwan were sub-optimally managed, as suggested by the less than ideal adherence data.

A study of 341 Parkinson disease patients in the UK showed that those who were on ropirinole ER remained on treatment longer than patients on ropirinole IR, and those who were on ropirinole ER had better adherence [21]. We also found pramiprexole ER initiators having better adherence than pramipexole IR initiators during the pre-pramipexole ER era. However, the MPRs for pramipexole IR initiators from 2012 through 2016 were not directly comparable to those for pramipexole ER initiators during the same period, as switching from pramipexole IR to pramipexole ER could contribute to lower MPR.

Although no formal statistical evaluations were carried out in this report, the source data covered almost every resident in Taiwan and there was no statistical sampling or variation in the data. In the report by Beau-Lejdstrom that described the use of drugs for ADHD in the UK from 1992 through 2013, no statistical tests were performed [4]. Interestingly, the investigators reported that after the introduction of ER methylphenidate in the UK, use of methylphenidate IR was stable over subsequent years and use of ER methylphenidate did not increase to peak level until 4 years after introduction, an IR/ER use pattern that was similar to what we observed for pramipexole in Taiwan.

Secular trends of anti-Parkinson drug use have been evaluated in Japan [22] and New Zealand [23]. The national trend of anti-Parkinson drug use in Taiwan from 2004 through 2011 was reported in 2016, using the same data source as in this report [24]. The focus of the prior reports was on all anti-Parkinson drugs, rather than evaluating the impact of the introduction of a specific product.

\subsection{Limitations}

In Taiwan, ER formulations of levodopa/benserazide combination and levodopa/carbidopa combination have been approved for use for more than a decade before the study period. Long-acting biperiden and transdermal rotigotine patch were approved 2008, and a prolonged-release tablet of ropinirole was approved at the end of 2009. Due to limited resources and the objectives of evaluating recent data that would be most relevant for current drug use, we did not evaluate the impact of these ER formulations in this study. Duration of pramipexole ER and IR use for individual patients, switching patterns between pramipexole IR and pramipexole ER, and factors associated with switching are important research topics related to the objectives of the study, but they were not evaluated in this report.

As a study based on health-insurance claims, Parkinson's disease diagnosis codes may not represent the underlying disease conditions for all subjects, but requirement for both diagnosis and use of pramipexole should provide assurance that a vast majority of patients did have Parkinson's disease. In addition, there was no information on lifestyle factors, including smoking and alcohol consumption, which is a common limitation for studies based on these types of data. Another limitation of the study is that management of chronic diseases varies across countries, as a result of different health systems, health beliefs, and practice patterns. The findings were accurate descriptions of pramipexole use in Taiwan, but would not be generalizable to all other countries.

\section{Conclusion}

This population-based real-world study showed that introduction of pramipexole ER to Taiwan resulted in higher LEDDs in prescriptions with pramipexole. Patients with Parkinson's disease who were initiated on pramipexole ER had better adherence to the medication that those who were prescribed pramipexole IR. These favorable impacts of pramipexole ER after its introduction may translate to better patient outcomes.

Acknowledgements Data in this study were provided by the Health and Welfare Data Science Center of the Department of Statistics, Ministry of Health and Welfare, Taipei, Taiwan. The authors would like to thank Yen-Yun Yang for data development and analysis support and Yu-Tai Liu for compiling drug codes.

\section{Declarations}

Funding The study was supported by the National Taiwan University (08SZA32502, 08SZA32501, 108TZA32, 108L104071) and Boehringer-Ingelheim Taiwan (contract number 412850) as an investigator initiated non-interventional study. The funding sources played no role in the execution, analysis, and interpretation of findings.

Conflicts of interest/Competing interests $\mathrm{K}$ Arnold Chan was the principal investigator for several industry-sponsored studies during the design and execution of this study. Boehringer-Ingelheim is the manufacturer of pramipexole, the drug of interest in this study. The study team discussed with Boehringer-Ingelheim scientists the concept of the study before protocol development, but Boehringer-Ingelheim did not have input into the final study design, analysis, and interpretation of findings. Yu-Chun Hsieh and Shu-Feng Hsieh were research staff at the National Taiwan University Health Data Research Center and have no conflicts of interest to declare. Rou-Shayn Chen has served as a consultant and speaker for Boehringer-Ingelheim but has no conflicts 
of interest relevant to the content of this article and there are no other conflicts of interest to disclose.

Ethics approval The study was approved by the Research Ethics Committee of National Taiwan University Hospital (approval document number $201901080 \mathrm{~W}$ ). De-identified data were used and there was no contact with individual subjects.

Consent to participate Requirement for individual consent was waived.

Consent for publication Requirement for individual consent was waived.

Availability of data and material Per Taiwan government policy, source data and analytic data sets are kept at the Department of Statistics, Ministry of Health and Welfare and are not available for sharing with the research community.

Code availability SAS codes for data development and analysis are available for review.

Authors' contributions The research concept was first proposed by Rou-Shayn Chen. K. Arnold Chan and Rou-Shayn Chen designed the study and prepared the protocol with assistance from Shu-Feng Hsieh. K. Arnold Chan and Rou-Shayn Chen applied for funding from sponsors, with K. Arnold Chan as the principal investigator. Yu-Chun Hsieh and Shu-Feng Hsieh supported project management, data development, analysis, and preparation of the report. K. Arnold Chan wrote the article.

Open Access This article is licensed under a Creative Commons Attribution-NonCommercial 4.0 International License, which permits any non-commercial use, sharing, adaptation, distribution and reproduction in any medium or format, as long as you give appropriate credit to the original author(s) and the source, provide a link to the Creative Commons licence, and indicate if changes were made. The images or other third party material in this article are included in the article's Creative Commons licence, unless indicated otherwise in a credit line to the material. If material is not included in the article's Creative Commons licence and your intended use is not permitted by statutory regulation or exceeds the permitted use, you will need to obtain permission directly from the copyright holder. To view a copy of this licence, visit http://creativecommons.org/licenses/by-nc/4.0/.

\section{References}

1. Patil Kapil, Patil Prashant, Patil Javesh, Pawar Sunil. A basic approach on sustained release drug delivery system. Am J PharmTech Res. 2012;2:213-31.

2. Perrie Yvonne, Rades Thomas. Pharmaceutics: drug delivery and targeting. 2nd ed. London: Pharmaceutical Press; 2012.

3. Ding H. Modified-release drug products and drug devices. In: Shargel L, Yu AC, editors. Applied biopharmaceutics \& pharmacokinetics, 7 e. New York: McGraw-Hill; 2016. http://accesspharmacy.mhmedical.com/content.aspx?booki $\mathrm{d}=1592 \&$ sectionid=100674031. Accessed 14 May 2020.

4. Beau-Lejdstrom R, Douglas I, Evans SJ, Smeeth L. Latest trends in ADHD drug prescribing patterns in children in the UK: prevalence, incidence and persistence. BMJ Open. 2016;6:e010508.

5. Piercey MF. Pharmacology of pramipexole, a dopamine D-3-preferring agonist useful in treating Parkinson's disease. Clin Neuropharmacol. 1998;21:141-51.
6. Center for drug evaluation and research. In: U.S. Food and Drug Administration. https://www.accessdata.fda.gov/drugsatfda_docs/ nda/98/020667a_appltr.pdf. Accessed 15 May 2020.

7. Mirapexin. In: European Medicines Agency. https://www.ema. europa.eu/en/medicines/human/EPAR/mirapexin. Accessed 15 May 2020.

8. Poewe W, Rascol O, Barone P, Hauser RA, Mizuno Y, Haaksma $\mathrm{M}$, et al. Extended-release pramipexole in early Parkinson disease: a 33-week randomized controlled trial. Neurology. 2011;77:759-66.

9. Mizuno Y, Yamamoto M, Kuno S, Hasegawa K, Hattori N, Kagimura T, et al. Efficacy and safety of extended- versus immediate-release pramipexole in Japanese patients with advanced and L-dopa-undertreated Parkinson disease: a double-blind, randomized trial. Clin Neuropharmacol. 2012;35:174-81.

10. Wang Y, Sun S, Zhu S, Liu C, Liu Y, Di Q, et al. The efficacy and safety of pramipexole ER versus IR in Chinese patients with Parkinson's disease: a randomized, double-blind, double-dummy, parallel-group study. Transl Neurodegener. 2014;3:11.

11. Shen T, Ye R, Zhang B. Efficacy and safety of pramipexole extended-release in Parkinson's disease: a review based on meta-analysis of randomized controlled trials. Eur J Neurol. 2017;24:835-43.

12. Pellicano C, Benincasa D, Fanciulli A, Latino P, Giovannelli M, Pontieri FE. The impact of extended release dopamine agonists on prescribing patterns for therapy of early Parkinson's disease: an observational study. Eur J Med Res. 2013;18:60.

13. Tomlinson CL, Stowe R, Patel S, Rick C, Gray R, Clarke CE. Systematic review of levodopa dose equivalency reporting in Parkinson's disease. Movem Disord. 2010;25:2649-53.

14. NHI profile. In: National Health Insurance Administration Ministry of Health and Welfare. https://www.nhi.gov.tw/english/Conte nt_List.aspx $? \mathrm{n}=8 \mathrm{FC} 0974 \mathrm{BBFEFA56D \& topn=ED4A30E51A}$ 609E49. Accessed 15 May 2020.

15. Hsing AW, Ioannidis JP. Nationwide population science: lessons from the Taiwan National Health Insurance Research Database. JAMA Intern Med. 2015;175:1527-9.

16. Lin LY, Warren-Gash C, Smeeth L, Chen PC. Data resource profile: the National Health Insurance Research Database (NHIRD). Epidemiol Health. 2018;40:e2018062.

17. Lin HM, Juan YC, Chen CD, Chan KA. Drug list compilation for pharmacoepidemiology studies using available public resource in Taiwan. (in Chinese) Taiwan J Public Health. 2017;36:521-529.

18. Andrade SE, Kahler KH, Frech F, Chan KA. Methods for evaluation of medication adherence and persistence using automated databases. Pharmacoepidemiol Drug Saf. 2006;15:565-74.

19. Grosset D, Antonini A, Canesi M, Pezzoli G, Lees A, Shaw K, et al. Adherence to antiparkinson medication in a multicenter European study. Mov Disord. 2009;24:826-32.

20. Phillips LS, Branch WT, Cook CB, Doyle JP, El-Kebbi IM, Gallina DL, et al. Clinical inertia. Ann Intern Med. 2001;135:825-34.

21. Gungabissoon U, Kirichek O, Baou CE, Galwey N. Comparison of long-term use of prolonged-release ropinirole and immediate-release dopamine agonists in an observational study in patients with Parkinson's disease. Pharmacoepidemiol Drug Saf. 2020;29:591-8.

22. Nakaoka S, Ishizaki $\mathrm{T}$, Urushihara $\mathrm{H}$, Satoh $\mathrm{T}$, Ikeda $\mathrm{S}$, Yamamoto $\mathrm{M}$, et al. Prescribing pattern of Anti-Parkinson drugs in Japan: a trend analysis from 2005 to 2010. PLoS One. 2014;9:e99021.

23. Pitcher TL, MacAskill MR, Anderson TJ. Trends in antiparkinsonian medication use in New Zealand: 1995-2011. Parkinsons Dis. 2014;2014:379431.

24. Liu WM, Wu RM, Chang CH, Lin JW, Liu YC, Lin CH. National trends of antiparkinsonism treatment in Taiwan: 2004-2011. Parkinsons Dis. 2016;2016:1859321. 